\title{
ANALISIS DEL RIESGO Y LA PRIMA EN EL SEGURO AUTOMOTOR DURANTE LA PANDEMIA
}

\section{Hernández, Joaquín Alejandro}

\section{Resumen:}

La declaración de pandemia de la enfermedad Covid-19 por la O.M.S obligó a numerosos países a adoptar medidas preventivas de confinamiento en miras de proteger la salud y la vida; produciéndose a su vez un fuerte impacto en la economía global.

Al igual que la enfermedad, a nivel global proliferaron fake news, divulgadas con el fin de promover el pánico, confusión o conductas incorrectas entre los ciudadanos; motivando a la O.M.S a alertar sobre sus efectos perjudiciales, nombrando a este fenómeno como infodemia.

Argentina, al igual que numerosos países, adoptó medidas en favor del confinamiento social que implicaron la reducción de la interacción social y una significativa caída de la actividad económica.

El mercado asegurador argentino, no fue ajeno a los impactos sociales y económicos generados por la pandemia, como así tampoco a los nocivos efectos causados por infodemia.

A través de una primera fakenews, se indicó que los aseguradores del ramo automotor no cubrirían los siniestros de asegurados que violen el confinamiento.

Posteriormente, a través de otra, en razón de haberse producido una supuesta disminución del riesgo durante el confinamiento, se instó a los tomadores a solicitar masivamente descuentos en el precio del seguro, y se acusó a los aseguradores de haberse enriquecido sin causa.

En el presente artículo analizaremos las características del riesgo y la prima en el seguro automotor, demostrando que no existió disminución del riesgo durante el confinamiento y los peligros que conllevan la inobservancia de las bases técnicas del contrato de seguro.

Palabras claves: Argentina - Bases técnicas del seguro - Pandemia - Prima - Riesgo

- Seguro Automotor 


\section{Abstract:}

The declaration of pandemic Covid-19 disease by the O.M.S forced several countries to adopt preventive measures of confinement in order to protect health and life; producing a strong impact on the global economy.

Like the disease, fake news proliferated globally, spread in order to promote panic, confusion or misconduct between the citizens; what motivated the O.M.S to alert about its harmful and naming this phenom as infodemic.

Argentina, like many countries, adopted measures in favor of social confinement; these measures implied the reduction of social interaction and a significant drop in economic activity.

The Argentine insurance market also suffered several impacts because of the pandemic and the harmful effects caused by the infodemic.

In reference to the infodemic issue, there were two significative fake newe related to the pandemic and the auto insurance. The first one one tried to establish the idea that insurance companies will not cover the claims of policyholders who have violated the confinement.

The second one, supported by the reason of having suffered an alleged decrease in risk during confinement, encouraged the policyholders to request mass discounts in the price of the insurance, and the insurers were accused of enriching without cause.

In this article, we will analyze the characteristics of the risk and the premium in Argentine auto insurance, demonstrating that it has not existed a decrease of the risk during the confinement and which are the dangers and consequences of not observing the technical bases of the insurance contract.

Palabras claves: Argentina - Technical basis of insurance - Pandemic - Cousin Risk - Automotive Insurance

\section{PANDEMIA E INFODEMIA}

Desde que en fecha 11 de marzo de 2020 la Organización Mundial de Salud (OMS) declaró como pandemia a la enfermedad Covid-19 causada por el coronavirus SARSCoV-2, la humanidad entera se vio sensible y transversalmente afectada.

La importancia de la prevención, tantas veces menospreciada y postergada, irrumpió en nuestras vidas reivindicada con un valor preponderante, dado que ante una tragedia de proporciones globales, hasta el propio mercado y la economía debieron excepcionalmente- replegarse en función de la prioritaria y elemental necesidad de preservar la salud y la vida. 
Fue así que en aras de prevenir, la gran mayoría de los países -algunos antes que otros- adoptaron medidas de confinamiento a fin de evitar la propagación de la enfermedad, reduciéndose la interacción social y actividades económicas a sus niveles más básicos, con el único afán de permitir, con el menor daño posible, la simple supervivencia.

Nuestro país no fue la excepción a esta clase de medidas, dado que el Poder Ejecutivo Nacional, casi concomitantemente a la declaración de pandemia por la O.M.S, mediante el dictado del Decreto de Necesidad y Urgencia (en adelante denominado D.N.U) $\mathrm{N}^{\circ} 260$ de fecha 12 de marzo de 2020, amplió por un año la vigencia de la Ley de Emergencia Pública Sanitaria $N^{\circ} 27.541$, y estableció posteriormente, en fecha 19 de marzo de 2020, mediante el D.N.U N 297/2020, un aislamiento social, preventivo y obligatorio (en adelante denominado A.S.P.O), que exhortaba a las personas a permanecer en sus hogares, salvo honrosas excepciones autorizadas para el desempeño de actividades consideradas esenciales, que serían paulatina y progresivamente autorizadas.

El primer gran impacto de la pandemia, propagado a niveles globales como una fuerte onda expansiva, no fueron las lamentables muertes y contagios contados por miles, ni menos aún las cuantiosas y significativas pérdidas económicas causadas por el confinamiento y reducción de actividad económica; sino el recuerdo de una enseñanza de fuente aristotélica que comprende la dimensión individual sólo en función de la vida en sociedad, reivindicando a esta última como el único medio que permite la supervivencia, al sostener que quién no puede vivir en sociedad, o no necesita nada por su propia suficiencia, no es un individuo, sino una bestia o un dios.

De esta manera, gran parte de la humanidad recordaría que el bienestar individual no puede ser alcanzado sin el cuidado y protección del bienestar ajeno, constituyéndose este último en una premisa fundamental, no ya para alcanzar determinados niveles o estándares de confort y desarrollo, sino para lograr la propia supervivencia.

No obstante ello, las bestias y los mundanos dioses no fueron desterrados, por lo que aún durante la pandemia, comenzaron a proliferar nuevamente ciertos fenómenos que causarían zozobra entre la población mundial.

Entre ellos, las noticias falsas (fake news) transmitidas por las virtuales venas de una sociedad tecnologizada, proliferaron, al igual que el virus, de tal manera que hasta la propia O.M.S se vio obligada a alertar a la población sobre sus efectos perjudiciales, bautizándolas -atinadamente- con un creativo neologismo: infodemia.

La difusión de estas noticias falsas, persigue el fin promover pánico, confusión o conductas incorrectas entre los ciudadanos, a fin de satisfacer los intereses 
individuales de quién las propaga; por lo que su difusión constituye, acciones ética, moral y legalmente reprochables.

Dentro de este contexto, la pandemia y la infodemia, irrumpieron en la sociedad poniendo a prueba sus bases.

\section{PANDEMIA E INFODEMIA EN EL MERCADO ASEGURADOR}

Naturalmente, el brutal impacto social y económico que la pandemia generó a escala global, tuvo su correlato en el sector asegurador argentino, siendo afectado en múltiples aspectos. No siendo el objeto del presente trabajo analizar cada uno de ellos, enunciaremos sólo algunos.

Producto del confinamiento y paralización de gran parte de la actividad económica, no sólo la operatoria normal y habitual de las compañías aseguradoras argentinas se vio restringida, sino que además, aparecieron serias y preocupantes dificultades en la percepción de primas, producto de la disminución de la capacidad de los tomadores de contratos para hacer frente a sus obligaciones.

Asimismo, producto de un fenomenal desplome de los activos financieros a nivel local -aún más profundo que el sucedido a nivel global-, el patrimonio de las aseguradoras argentinas fue duramente golpeado, poniendo a prueba la capacidad de respuesta de múltiples entidades, que de verse compelidas a realizar anticipadamente esos activos, sufrirían cuantiosas e irremediables pérdidas.

Al respecto, no resulta ninguna novedad en el mercado asegurador argentino, la existencia de resultados técnicos negativos en la operatoria de determinados ramos (ej. Automotor) que luego son compensados con resultados financieros positivos, que producto del fenómeno mencionado, repentinamente se esfumaron.

Al ritmo de la paralización de la actividad económica y el consecuente impacto en los ingresos de la población, también aumentó la preocupación de los aseguradores respecto la sostenibilidad de la comercialización de sus productos y servicios, en virtud de la conocida y estrecha vinculación existente entre ambas.

Dentro de este contexto, la infodemia tampoco rehusó su ingrata presencia a los lares del mercado asegurador argentino, atacando uno de sus flancos más conocidos: el ramo del seguro automotor.

La primera fake news proliferó a gran escala por las redes sociales alertando a la población de que, en caso de sufrir un siniestro en ocasión de circulación con vehículos, en violación al A.S.P.O dispuesto por el D.N.U N²97/2020, la cobertura contra la responsabilidad civil sería rechazada por las compañías aseguradoras, en tanto constituir una supuesta exclusión de cobertura. 
La segunda fake news, de idéntica difusión, alertó a la población de que, en razón de la disminución de la circulación de vehículos producto del cumplimiento del confinamiento dispuesto por el mismo decreto, las compañías aseguradoras deberían otorgar descuentos en las primas, por haberse producido una supuesta disminución del riesgo y consecuente baja de siniestralidad, que enriquecería sin causa a las aseguradoras que obtendrían ganancias extraordinarias no previstas al momento de contratar.

Contra ambas fake news, se levantaron autorizadas voces del derecho nacional y hasta el propio Organismo de Contralor Estatal de la actividad aseguradora, habiendo la Superintendencia de Seguros de la Nación (SSN) emitido dos comunicados informando a la sociedad que, en el primer caso, no existía normativa alguna que autorizara a una compañía de seguros a rechazar la cobertura asegurativa ante la violación del A.S.P.O; y en el segundo caso, que tampoco existía normativa alguna que las obligara a realizar descuentos o bonificaciones a los asegurados.

No obstante ello, enroladas detrás de esta segunda fake news, numerosas voces cantantes y sonantes se alzaron enarbolando las banderas de una cruzada librada contra las "ganancias extraordinarias" del sector asegurador argentino y en "favor de los derechos de los asegurados"; hasta el punto de llegar a difundirse por medios de comunicación masiva, formularios pro forma instando a los tomadores a solicitar descuentos a sus aseguradores.

Por si fuera poco, también se anunció -con el infaltable disfraz altruista que de gala viste a la demagogia- la presentación de un proyecto de ley instado por un legislador, para obligar a los aseguradores argentinos a "adecuarse a la actual situación" mediante bajas sustanciales de primas.

Sin perjuicio de ello, numerosas compañías aseguradoras locales, a petición del algunos tomadores que exigían sus supuestos derechos, o bien manifestaban una merma en sus ingresos, comenzaron a otorgar descuentos de hasta el $50 \%$ de la prima anteriormente cotizada; planteándose así nuevos interrogantes sobre la capacidad de las aseguradoras argentinas para realizarlos y su impacto en el mercado.

\section{CARACTERISTICAS DEL RIESGO Y LA PRIMA EN EL CONTRATO DE SEGURO}

A fin de analizar adecuadamente las situaciones descriptas desde la óptica del Derecho del Seguro, es preciso abordar, previa y sintéticamente, algunas 
características propias del contrato de seguro que guardan íntima vinculación con la problemática planteada.

Conforme surge de una interpretación hermenéutica del microsistema de Derecho del Seguro, con el resto el ordenamiento jurídico positivo nacional, se desprende que el riesgo (objeto), junto con el consentimiento y el interés asegurable (causa), constituyen los elementos estructurales o esenciales del contrato de seguro, de manera que de faltar alguno de ellos, se afectaría la existencia y validez del contrato.

La ausencia de riesgo puede ser originaria, cuando se produce al momento de perfeccionamiento del contrato (por acaecimiento del siniestro o desaparición de la posibilidad de su ocurrencia), o derivada, cuando se produce cuando el contrato se encuentra en curso de ejecución.

En el primer caso, nos encontramos ante un supuesto inexistencia de riesgo que trae aparejada su nulidad; mientras que en el segundo supuesto, estamos ante un supuesto de cese del riesgo, siendo válido el contrato hasta el momento de su desaparición. Cuando el riesgo no desaparece, sino que se aminora, hablamos de disminución.

En tanto objeto del contrato de seguro, el riesgo estará determinado por la probabilidad o posibilidad de realización o producción del evento dañoso (siniestro), sobre el cual el asegurador brindó cobertura, contra el pago de la prima o cotización a cargo del tomador; debiendo la posibilidad de ocurrencia del riesgo ser incierta y además, futura.

El estado del riesgo es el conjunto de circunstancias relevantes y pertinentes para establecer la posibilidad o probabilidad siniestral. Al encontrarse el estado del riesgo dentro de la esfera de conocimiento y control del asegurado -dado que este no transfiere el riesgo en sí mismo sino las consecuencias económicamente disvaliosas derivadas de su eventual producción- es carga del tomador comunicar al asegurador todas aquellas circunstancias que lo constituyan de manera previa a la celebración del contrato, y las que lo agraven, disminuyan o lo extingan, con posterioridad a su celebración.

La ley de Contrato de Seguro (en adelante L.S), prevé en su Título I, Capítulo I, Sección II (Arts. 5 a 10) remedios para aquellos supuestos en que el asegurado no denuncia el verdadero estado del riesgo al tiempo de su perfeccionamiento, regulándose el instituto de la reticencia; precisando a su vez en el Título I, Capítulo I, Sección X (Arts. 37 a 45) los supuestos de agravación del riesgo producidos con posterioridad a su celebración.

Para que la agravación del riesgo se produzca y cause efectos en el contrato, se requiere que sobrevengan elementos objetivos o subjetivos. Los elementos objetivos 
se verificarán sobre el estado del riesgo si sobreviene una alteración sostenida y trascendente que aumente la probabilidad o la intensidad del riesgo tomado a cargo por el asegurador.

Respecto a los elementos subjetivos que pueden producir una agravación del riesgo, conforme enseña el ilustre maestro, Dr. Rubén S. Stiglitz, se requiere que "sobrevenga una alteración de las condiciones subjetivas del asegurado que sirvieron al asegurador para formarse opinión del estado del riesgo al concluir el contrato, debido a un hecho nuevo, no previsto ni previsible, relevante e influyente, que de haber existido al tiempo de concertarse el contrato habría impedido su celebración o incidido para que no se formalizara en las mismas condiciones" (2016: 504).

La L.S regula en su Art. $34^{\circ}$ párr. $1^{\circ}$ el derecho del tomador a obtener una rectificación parcial de la prima, en el supuesto de haber denunciado por error un estado del riesgo más grave, siendo este último un supuesto diferente al de cese o disminución del riesgo, dado que el mismo nunca existió.

Respecto a la disminución del estado del riesgo, que lógicamente debe suscitarse durante el transcurso del contrato, la L.S establece en su Art. $34,2^{\circ}$ párr. que "cuando el riesgo ha disminuido, el asegurado tiene derecho al reajuste de la prima por los períodos posteriores, de acuerdo a la tarifa aplicable al tiempo de la denuncia de la disminución".

Para su configuración, se requieren exactamente los mismos elementos y requisitos, que los enunciados para la agravación del riesgo.

La prima es la contraprestación de la obligación del asegurador, a cargo del tomador. En la estructura del precio del seguro, la prima pura es el valor actuarial del riesgo calculado por el asegurador. La prima pura más los costos de la operación, gastos de adquisición, explotación y la posible utilidad, constituyen la prima de tarifa, que sumada a los impuestos y tasas, constituyen al premio del seguro o prima bruta; debiendo todos los conceptos estar discriminados en las pólizas emitidas por el asegurador.

Debe destacarse la íntima vinculación que existe entre el riesgo asumido por el asegurador y el pago de le prima pura abonada por el tomador, debiendo observarse entre el objeto de ambas obligaciones una estrecha relación de equivalencia que preserva el equilibrio de las prestaciones.

Por ser mencionada relación el objeto de análisis del presente, al abordar en adelante el concepto de prima, nos referiremos a la prima pura.

Para la determinación o cálculo de la prima por parte del asegurador, se deben observar los criterios de suficiencia, equidad, correlatividad y representatividad. 
La suficiencia está referida a la necesidad de que la tarifa cubra razonablemente la tasa de riesgo (prima pura) más los costos de la operación, gastos de adquisición, explotación y la posible utilidad (prima de tarifa).

Si bien los aseguradores pueden fijar libremente las tarifas de las primas, deberán resguardar que las mismas sean suficientes para cumplir con las obligaciones asumidas (cobertura de siniestros), dado que, de lo contrario, se pondría en grave riesgo su operatoria.

Por su parte, el Organismo de Contralor Estatal de la actividad aseguradora, en ejercicio de su poder de policía, deberá velar por el mantenimiento de la referida suficiencia. (Art. $\mathrm{N}^{\circ} 26$, Ley argentina de Entidades de Seguros y su Control, $\mathrm{N}^{\circ}$ 20.091)

La equidad en la fijación de la prima impone que su determinación no puede ser abusiva, ni discriminatoria.

La obligación de observación respecto a que la prima y el riesgo mantengan una correlación positiva, corresponde al asegurador, verificándose así el principio de correspectividad a los fines de preservar el equilibrio prestacional prima/riesgo.

La homogeneidad requiere que los elementos de la muestra sobre cual se calcula deban tener características comunes en los órdenes cualitativos y cuantitativos; debiendo además la muestra ser representativa de la masa de riesgos que se asumen. Por disposición legal, respetando los principios enunciados, el cálculo de la prima es realizada por el asegurador, entre otros, en base a la experiencia siniestral (Art, 26.1.2 inc. b) Reglamento General de la Actividad Aseguradora en Argentina) es decir, en base a estadísticas pasadas, proyectadas hacia el futuro, con basamento en la ley de los grandes números.

La llamada ley de los grandes números, es una teoría de la probabilidad que refiere a que los acontecimientos eventuales y semejantes, observados experimental y repetitivamente con tendencia al infinito, estadísticamente decrecen en su irregularidad hasta generar una constante.

En base a esta constante, obtenida de la observación empírica de la frecuencia e intensidad de siniestros sobre categorías de riesgo homogéneas, el asegurador, puede prever y proyectar -remarcamos- a futuro, los siniestros que sufrirán parte de la mutualidad de asegurados, y distribuir entre ella el costo del seguro mediante una tasa de prima que será aplicada sobre la suma asegurada de cada contrato, constituyendo así un fondo de primas para hacer frente a los siniestros. Es por ello que se dice que el contrato de seguro reposa sobre bases técnicas.

La lógica del negocio técnico del seguro, implica que cada contrato, más allá de su aparente individualidad, se encuentre conectado con una masa de contratos 
semejantes que permitan la distribución del riesgo entre la masa de asegurados, sin la cual la dinámica asegurativa no podría llevarse a cabo, ni diferiría en su naturaleza jurídica de un mero contrato de apuesta.

En el mercado asegurador, establecer la exposición a riesgo en cada contrato particular resulta una cuestión de dificultosa realización fáctica (ej. saber cuántos kilómetros recorre un vehículo durante la vigencia de una póliza) por lo que el precio de un contrato seguro, individualmente considerado, no estará determinado por el riesgo en sí mismo, sino por un porcentaje de la constante de riesgo proyectada para la mutualidad de asegurados; previamente calculada mediante ley de los grandes números y en base a categorías de análisis de riesgo homogéneas.

La prima se encuentra además regida por el principio de invariabilidad que indica que la misma no puede alterarse, salvo acuerdo de partes o disposición contenida en una norma legal; no pudiendo, en el resto de los supuestos, ser modificada.

Los supuestos de variación de la prima contemplados en la L.S.A, son la reticencia no dolosa (Art. 6 LS), la agravación del riesgo (Art. 35 LS) y disminución del riesgo (Art. 34 LS).

Para que la denuncia del agravamiento o disminución del riesgo puedan tener incidencia en un reajuste de la prima, su denuncia deberá versar sobre aquellos parámetros objetivos o subjetivos evaluados por el asegurador a los fines de analizar el riesgo.

\section{REFLEXIONES SOBRE EL RIESGO EN EL SEGURO AUTOMOTOR DURANTE LA PANDEMIA Y SU INCIDENCIA EN EL PRECIO DEL SEGURO.}

El contrato de seguro automotor, conforme surge de nuestro ordenamiento jurídico argentino, posee una causa constituida por el interés económico-lícito del asegurado sobre el mantenimiento de su indemnidad patrimonial (interés asegurable), sea por cuanto este deba a un tercero en razón de los acontecimientos previstos en la cobertura contra la responsabilidad civil, o bien, sobre el detrimento que sufra en el bien integrante de su patrimonio (automotor) en la cobertura de casco (incendio, robo, daño, etc.); constituyendo éstos, a su vez, los riesgos asumidos por el asegurador (objeto), mediante la percepción del pago de la prima.

La mera existencia y finalidad de la cobertura contra la responsabilidad civil supone necesariamente la violación a normas jurídicas por parte del asegurado (deber no dañar a otro, normas de tránsito, etc.) dado que, sin la previa violación de esas disposiciones legales, el siniestro no podría producirse.

La necesidad de que la cobertura proceda ante violación de mandatos legales, no implica que el riesgo asumido no pueda estar delimitado objetivamente mediante 
exclusiones de cobertura, que contenidas en el contrato, constituyen supuestos de no seguro; o delimitaciones subjetivas relacionadas al dolo y la culpa grave, que acarreen la caducidad del derecho del asegurado a ser indemnizado (Arts. 70 y 114 LSA).

La mera circulación en violación al A.S.P.O ordenado en Argentina mediante del DNU $N^{\circ} 297 / 2020$ no se encuentra inmersa ninguna delimitación objetiva ni subjetiva del riesgo asumido por el asegurador, y por lo tanto, su verificación no lo exonera de brindar cobertura a los siniestros que se produzcan dentro de los límites del contrato (Art. 118 LSA).

La violación de lo dispuesto por el D.N.U N²97/2020 podrá acarrear sanciones administrativas o penales al infractor, de la misma manera que pueden implicar idénticas sanciones, el por ej. cruzar un semáforo en rojo, o bien, lesionar o matar a un peatón; sin que las referidas circunstancias enerven la procedencia de la cobertura.

Desmentida mencionada cuestión, aparecida durante la pandemia como una primer fake news relacionada al contrato de seguro automotor, debemos adentrarnos en el análisis de la segunda, consistente en la circulación de la noticia de que las compañías aseguradoras argentinas deberían otorgar descuentos en las primas percibidas, por haberse producido una supuesta disminución del riesgo y consecuente baja de siniestralidad durante el confinamiento, conllevado un enriquecimiento sin causa de las mismas.

Cabe entonces preguntarse si la disminución en la circulación de vehículos, producto del A.S.P.O ordenado mediante del DNU N²97/2020 y, consecuente baja de siniestralidad observada con posterioridad, otorga derecho a los tomadores de seguros a reclamar a sus aseguradores una reducción de la prima en base a una supuesta disminución del riesgo.

Para ello, deberemos analizar las características del riesgo y cálculo de la prima en este contrato en particular, y su eventual afectación mediante las circunstancias de hecho producidas a raíz de la pandemia.

Supuesto de interrupción de la circulación:

Conforme lo señalamos anteriormente, el riesgo en el contrato de seguro estará determinado probabilidad o posibilidad de realización o producción del evento dañoso, debiendo además estar previsto en el contrato.

De la circunstancia que, como señalamos en la crítica a la primer fake news, la cobertura asegurativa se mantendría respecto de los siniestros ocurridos en ocasión de circulación en violación al A.S.P.O, se desprende que la posibilidad de ocurrencia del riesgo previsto en la cobertura contra la responsabilidad civil, no se ha visto alterada por el dictado del D.N.U 297/2020. 
Atento a la subsistencia de la posibilidad de ocurrencia de un siniestro acaecido en violación a lo dispuesto por la referida normativa, si un asegurado hubiera decidido cumplir estrictamente con el dictado de mencionada norma y dejar de circular; respecto la cobertura contra la responsabilidad civil, se produciría un supuesto de cese del riesgo, que por imperativo legal debe ser denunciado al asegurador al momento de su conocimiento, adquiriendo el tomador derecho a reducción de la prima (en caso mantenerse las coberturas de casco) o bien rescindir el contrato con derecho a la devolución de las primas; en ambos casos, correspondientes a los períodos abonados posterioridad a su comunicación.

En ningún supuesto, la reducción o devolución de la prima en base a un cese del riesgo, podrá ser válidamente reclamada respecto de períodos transcurridos sin la previa comunicación al asegurador, dado que al encontrarse el estado del riesgo en la esfera de conocimiento del asegurado, el tomador sufrirá las consecuencias del incumplimiento de la carga de información.

\section{Supuesto de disminución de circulación:}

Evaluada la hipótesis de cese del riesgo, cabe analizar ahora el supuesto en que el tomador o asegurado ha decidido no interrumpir, sino disminuir su circulación, y si mencionada circunstancia puede ser considerada una disminución del riesgo que otorgue derecho a una reducción de la prima en los términos del Art. 34 párr. $2^{\circ}$ LS.

Al igual que en los supuestos de agravación del riesgo, como enunciamos, sobrevenidos con posterioridad al perfeccionamiento del contrato, para que pueda considerarse que ha habido una disminución del riesgo, se requiere que sobrevengan elementos objetivos o subjetivos.

Los elementos objetivos se verificarán sobre el estado del riesgo, si sobreviene una alteración sostenida y trascendente que aumente la probabilidad o la intensidad del riesgo tomado a cargo por el asegurador.

A su vez, podrá considerarse que hubo una disminución del riesgo, si con posterioridad a la celebración, sobreviene una alteración de las condiciones subjetivas del asegurado que sirvieron al asegurador para formarse opinión del estado del riesgo al momento celebrar el contrato.

En ambos supuestos, la disminución del riesgo debe darse en base a un hecho nuevo, no previsto, ni previsible, debiendo ser relevante e influyente, a tal punto que de haber existido al tiempo de celebrarse el contrato, habría impedido su celebración o incidido para que no se formalizara en las mismas condiciones.

En el mercado asegurador, salvo excepcionales supuestos de seguro automotor contratados mediante el sistema de scoring o segmentación de riesgos, los kilómetros recorridos por un rodado individualmente considerado, no son circunstancias 
relevantes consideradas por el asegurador a los efectos de analizar el riesgo y establecer la prima correspectiva.

Al momento de contratación del seguro, el tomador es consultado sobre la zona de circulación, marca, modelo y año del rodado, la propulsión del mismo, uso comercial o particular, etc.; pero no sobre cuántos kilómetros recorrería, ni si el mismo será utilizado en forma permanente o esporádica.

En razón del fenómeno de cálculo de la tasa de la prima sobre una constante obtenida mediante la ley de los grandes números, el asegurador argentino no considera, ni siquiera generalmente, el tiempo o distancia de utilización del rodado como una categoría homogénea para analizar el riesgo.

En rigor, podría suceder que un asegurado utilice el rodado una vez al mes, y otro, lo realice las $24 \mathrm{hs}$. de todos los días, abonando ambos tomadores exactamente la misma tasa de prima respecto la cobertura contra la responsabilidad civil contenida en la póliza.

Atento a que el riesgo de producción de un siniestro es incierto, no se observa tampoco -al menos con una base actuarial cierta y verificable- una disminución de la posibilidad de ocurrencia de un siniestro en base a la disminución de circulación, de manera que permita sostener, por ej. que un vehículo que circule $200 \mathrm{~km}$ por la escasamente poblada llanura pampeana, tenga más probabilidades de sufrir un siniestro que otro que circula $100 \mathrm{~m}$. en el centro de la ciudad de Buenos Aires; o que este último tenga una menor probabilidad de sufrir un siniestro de magnitud, respecto de otro que circula $10 \mathrm{~km}$ por la misma ciudad.

Calcular la prima en relación al tiempo de utilización del rodado podría constituir una excelente idea a futuro para pólizas emitidas en el mercado asegurador argentino tomando en cuenta esa variable, máxime aun cuando en la actualidad las herramientas tecnológicas para medir la circulación de un rodado se encuentran literalmente- al alcance de la mano; y servirían no sólo para medir el tiempo y la distancia de circulación, sino también para brindar datos que permitan realizar certeramente cálculos actuariales.

No obstante, al no ser considerada esta circunstancia en la mayoría contratos de seguro automotor celebrados en nuestro país como un elemento relevante e influyente para el cálculo de la prima, por aplicación de la normativa de seguros foránea, no podrá ser alegada válidamente una supuesta disminución del riesgo que otorgue a los tomadores derecho a una reducción de la prima; ni siquiera aún en el hipotético supuesto de verificarse que la disminución en la circulación influye en la incidencia del riesgo como un factor coadyuvante. 
Al respecto, en un supuesto análogo de agravación del riesgo, el Tribunal Supremo de España expresó que "carecen de relevancia, en relación con el contrato de que se trata, circunstancias asimismo omitidas por el asegurado, como la de tratarse de un fumador antiguo y la de haber sido trabajador de minas de carbón, datos que no conllevan, en la mayor parte de los casos, la aparición de enfermedades oncológicas, como la que le produjo al mismo la muerte, siendo, en cualquier caso, meros factores de riesgo, que pueden coadyuvar o no, a la aparición de la enfermedad, por lo que el resaltarlos es incrementar una posible presunción que carece, en muchísimos casos, de certeza" (2004: 1681).

\section{Supuesto de disminución de la siniestralidad:}

Descartada la posibilidad de que en el mercado asegurador, el tomador o asegurado puedan argüir una disminución del riesgo con derecho a reducción de prima en base a una supuesta disminución de utilización del rodado individualmente considerada, cabe ahora analizar el argumento de que producto del dictado del A.S.P.O se ha producido una baja de la siniestralidad y consecuente enriquecimiento sin causa por parte delos aseguradores, otorgando al tomador el derecho a la reducción de la prima.

Conforme lo enunciamos, el asegurador, a los efectos de calcular la prima recurre remarcamos- previamente y en base a experiencias pasadas, a la ley de los grandes números a fin de realizar una previsión y proyección de la frecuencia e intensidad de los siniestros que pueden producirse a futuro; distribuyendo el riesgo que resulte de la constante proyectada en base a categorías homogéneas, entre la mutualidad de asegurados.

A los fines de analizar una hipotética disminución del riesgo producto de una supuesta baja de siniestralidad tras la implementación del A.S.P.O, no debe perderse de vista que el asegurador realiza las proyecciones de riesgos hacia el futuro y no desde el futuro.

Consecuentemente, los análisis sobre las proyecciones de riesgos realizados ex post, no son análisis de riesgos, sino de resultados, constituyendo circunstancias absoluta y diametralmente diferentes.

En este orden de ideas, implicaría un contrasentido, ajeno a la naturaleza del negocio celebrado, que el asegurador, tras un análisis ex post que arroje resultados negativos respecto de la frecuencia e intensidad de siniestros proyectados, exija a la masa de asegurados una contribución mayor para hacer frente a sus pérdidas, alegando que los tomadores se enriquecieron sin causa porque abonaron menos de lo que correspondía.

En relación, las eventuales pérdidas sufridas en ocasión de la menor cantidad e intensidad de siniestros proyectados, constituirán un riesgo propio de la operatoria del 
asegurador, siendo asumida y solucionada, no mediante la exigencia de mayores aportes a la masa de asegurados, sino a través del sistema de reaseguro.

Consecuentemente, debemos preguntarnos si al momento de la implementación del A.S.P.O el asegurador estuvo en condiciones de advertir la ocurrencia de una disminución del riesgo a futuro, y si la baja de siniestralidad observada con posterioridad, reúne los requisitos necesarios para ser considerada una disminución del riesgo que autorice a sus asegurados el derecho a reclamar una reducción de la prima.

Conforme lo establece Art. N961 del Código Civil y Comercial de la Nación, los contratos deben celebrarse, interpretarte y ejecutarse de buena fe, obligando a las partes con los alcances a que razonablemente se hubiese obligado un contratante cuidadoso y previsor.

El impacto causado por la enfermedad Covid-19 constituyó una circunstancia atípica y sin precedentes cercanos, no sólo en Argentina, sino en el mundo, que se produjo a una escala tal que colapsó hasta los sistemas sanitarios y económicos de los países de más alto índice de desarrollo humano (IDH).

Si mencionada situación, con directa incidencia en la vida y salubridad de la humanidad, no pudo ser prevista por los gobiernos y países más avanzados en términos de desarrollo, el exigirle a un asegurador argentino que haya previsto la incidencia que una pandemia tendría en la siniestralidad proyectada para el parque automotor de su país, resulta cuanto menos, una imputación excesivamente pretenciosa y ciertamente irrazonable, obligándonos, en consecuencia, a examinar en el grado de previsión exigido un criterio eminentemente restrictivo.

Debemos destacar que dentro del contexto señalado, el A.S.P.O dictado por el Poder Ejecutivo Nacional en fecha 19 de marzo de 2020, mediante D.N.U 297/2020, constituyó una medida excepcional y provisoria, prorrogada posteriormente de forma paulatina en base a decisiones de gobierno basadas en estudios epidemiológicos realizados en forma periódica. Asimismo, a medida que transcurrieron los días de su vigencia, fueron también aumentando significativamente las actividades y zonas del país exceptuadas mediante el dictado de Decisiones Administrativas emanadas en una primer etapa de la Jefatura de Gabinete de Ministros y posteriormente de los gobiernos provinciales; debiendo presuponerse, en razón de la disminución de impacto progresivo de la mencionada normativa, que si la siniestralidad en el mercado del seguro automotor eventualmente disminuyó a causa de la disposición de confinamiento dictada, también debería lógicamente aumentar a medida que la restricción era paulatinamente excepcionada. 
En razón de la imprevisibilidad de la supuesta disminución de siniestralidad causada por la medida, claramente no existió una experiencia previa que proporcione al asegurador bases certeras, de manera que le hayan permitido proyectar una hipotética baja de siniestralidad $y$ realizar los análisis actuariales correspondientes a los fines de calcular un eventual reajuste de la prima.

Asimismo, en virtud de la excepcionalidad, provisoriedad y disminución de impacto progresivo del A.S.P.O, tampoco se verifican elementos objetivos que permitan al asegurador haber realizado, ni realizar a futuro cálculos actuariales sobre una eventual e hipotética baja la probabilidad o intensidad de producción siniestros en base a categorías homogéneas previamente establecidas que puedan ser sostenidas en el tiempo.

Si la situación observada no permitió, en razón de su imprevisibilidad, ni permite a futuro, en razón de su provisoriedad, realizar el correspondiente análisis de riesgo en base a categorías homogéneas, previamente establecidas y que puedan sostenerse en el tiempo, consideramos que desde la perspectiva de la normativa de seguros no se reúnen los requisitos jurídicos necesarios para ser considerada una disminución del riesgo.

La baja de siniestralidad alegada por quienes reclamaron una reducción de primas, lejos de resultar un análisis de riesgos, constituye un análisis de resultados, que ni siquiera, en razón del estado de excepcionalidad y provisoriedad de las medidas de confinamiento dictadas, pueden ser proyectados con base cierta hacia el futuro.

La única certeza que le asiste al asegurador es que, aún durante la vigencia del A.S.P.O deberá hacer frente a los siniestros que se produzcan dentro de los límites del contrato, sea que los asegurados estén o no, respetando el A.S.P.O.

En razón de lo mencionado, aún de haberse producido, o de producirse una baja de la siniestralidad dentro del atípico, provisorio y variable contexto imperante durante la vigencia del A.S.P.O -al igual que en el supuesto anteriormente mencionado respecto la hipótesis de aumento de siniestralidad no prevista- constituiría un resultado ventajoso propio de la dinámica del negocio asegurador; sin que -al igual que la falta de derecho del asegurador para exigir mayores primas ante un resultado desfavorableotorgue derecho al tomador a exigir válidamente una reducción de las primas.

Demás está aclarar que la reducción o devolución de primas, bajo ningún concepto podrá según la Ley de Contrato de Seguro, realizarse en base a períodos de seguros transcurridos y con improcedentes fundamentos basados en análisis de resultados; dado que el asegurador adquirió el derecho al cobro de las mismas en razón del estado del riesgo que resultaba posible y razonablemente previsto mediante los 
cálculos actuariales que tenía a disposición, y desde el momento previo al que la siniestralidad pueda analizarse.

Lo que la Superintendencia de Seguros de la Nación (SSN) debió decir en su comunicado, no es que "no existe normativa alguna que obligue a las aseguradoras a aplicar descuentos", sino que la situación en virtud de la cual los mismos eran exigidos, no encuadraba dentro de la normativa que otorga al tomador derecho a solicitarlos, por no existir en términos jurídicos una disminución del riesgo.

Asimismo, en razón de la armónica convivencia y complementación que debe primar en el Derecho Positivo entre las disposiciones contenidas en el régimen especial de Derecho del Seguro y el general de Protección a Consumidores y Usuarios, se debe destacar que los postulados enunciados en el presente artículo no se contraponen con los principios, derechos y garantías propios del régimen consumeril.

Se debe también señalar que por disposición del Art. 1121 inc. a) del Código Civil y Comercial de la Nación Argentina, se encuentra vedada la posibilidad de declarar abusivas las cláusulas relativas a la relación entre el precio y el bien o servicio procurado.

\section{DESCUENTOS APLICADOS $Y$ RIESGOS DERIVADOS DE LA INOBSERVANCIA A LAS BASES TECNICAS DEL SEGURO.}

Dentro de este contexto, podemos sostener que los descuentos otorgados por algunas compañías aseguradoras argentinas a ciertos tomadores -que en ocasiones llegaron hasta el $50 \%$ de la prima- no tuvieron basamento en una disminución del riesgo, sino en la mera aplicación de políticas comerciales de las mismas, destinadas a retener clientes.

Si bien las Condiciones Generales de la Póliza Seguro Automotor son uniformes para todos los aseguradores argentinos, no lo son los cuadros tarifarios que cada de uno de ellos aplica y que deben ser previamente aprobados por la S.S.N en virtud de la capacidad económica financiera de cada asegurador.

El otorgamiento de descuentos en las primas por parte de los aseguradores no constituye en sí mismo una práctica ilegal, en tanto no se viole el principio de suficiencia de la prima dispuesto por el Art. 26 de la Ley argentina de Entidades de Seguros y su Control No 20.091.

De otorgar una asegurador descuentos por debajo de los estándares de suficiencia exigidos por la ley, no sólo podría incurrir en una práctica prohibida por la Ley de Defensa de la Competencia $N^{\circ} 27.442$, sino que además pondría en serio riesgo su 
capacidad de hacer frente a los siniestros que se produzcan, perjudicando a la mutualidad de asegurados mediante una práctica irregular de la actividad.

En virtud de lo dispuesto por el Art. $26^{\circ}$ Ley 20.091, será el Organismo de Contralor Estatal de la actividad aseguradora quien, en ejercicio de su poder de policía, vele por la suficiencia de las primas cobradas por las aseguradoras.

En caso de detectarse, deberán ser neutralizadas y sancionadas aquellas prácticas mediante las cuales los aseguradores otorguen descuentos en afectación su capacidad económica financiera; o bien constituyan acciones de dumping, consistentes en vender productos por debajo de su precio razonable a los fines de captar mercado mediante una competencia desleal y en perjuicio no sólo del mercado, sino, por sobre todo, de la mutualidad de asegurados.

La Superintendencia de Seguros de la Nación podrá también, en ejercicio de sus funciones, aprobar planes mínimos de tarifas cuando se halle en riesgo la estabilidad del mercado, sin que ello tenga correlación alguna con la hipotética e improcedente "disminución de riesgos" analizada.

La acción observada de arengar a los tomadores de seguros a lanzarse masivamente a solicitar descuentos convenciéndolos de poseer derechos de los que en realidad carecen resulta repudiable e imprudente.

Sostener que la eventual presencia un resultado positivo, producido dentro de la dinámica propia del negocio asegurador, constituye para un asegurador una ganancia extraordinaria, no sólo implica desconocer las reglas y principios jurídicos enunciados que rigen la actividad, sino también la preocupante y grave crisis que atraviesa el sector asegurador en nuestro país, conjuntamente con los principios más básicos y elementales que integran la contabilidad empresaria.

Con excepción de ciertos criterios utilizados en materia tributaria, no existen en nuestro Derecho elementos para evaluar la legitimidad o ilegitimidad de las ganancias obtenidas en un contrato en base a su cuantificación, sino reglas que deben ser observadas de buena fe para la realización lícita de negocios, y que en relación con la temática que abordamos, no se encontraron vulneradas ante la irrupción del estado de excepción.

La intención de obligar a las aseguradoras mediante ley a otorgar descuentos a sus asegurados en base a la hipotética disminución de riesgos y ganancias extraordinarias inexistentes resulta imprudente y atentatoria contra los principios y bases técnicas que rigen la actividad aseguradora.

Siguiendo la lógica aristotélica, así como en nuestra sociedad un individuo no puede ser considerado como un ser aislado y autosuficiente, requiriéndose del bienestar ajeno como base indispensable para la propia supervivencia y desarrollo individual y 
colectivo, en la dinámica del negocio asegurador, tampoco podrá considerarse a un contrato de seguro con independencia y autonomía de la mutualidad de asegurados que integraron el fondo de primas para hacer frente a los eventuales siniestros que se produzcan.

Atentar contra mencionado fondo de primas, como mediante la divulgación de fakes news que insten a los asegurados a solicitar descuentos masivamente, o bien intentar por ley que los aseguradores masivamente se desfinancien, constituye además una acción demagógica que producirá efectos perjudiciales sobre los intereses que "altruistamente" se declararon proteger a fin de legitimarlas.

Debe observarse y comprenderse que la actividad aseguradora reposa sobre bases técnicas sin las cuales no tendría razón de ser, repudiándose consecuentemente, todas aquellas acciones que presupongan que el asegurador pueda afrontar siniestros con los recursos inagotables propios de un dios; o que, mediante el dictado de normas jurídicas o pronunciamientos judiciales que no reconozcan la naturaleza de su operatoria, lo obliguen forzosamente a transformarse en una bestia.

La relación entre la prima y el riesgo, integrada por la adecuada observación de los principios enunciados, lleva ínsita una función de garantía tanto respecto de los derechos de la mutualidad de asegurados, como de la estabilidad del mercado.

Solamente comprendiendo y respetando la dinámica de funcionamiento del negocio asegurador, tanto en Argentina como en el mundo, se podrán instar acciones masivas, dictar leyes y emitir pronunciamientos judiciales que, en vez de debilitar la mutualidad de asegurados, la fortalezcan; y que, en lugar de atentar contra la subsistencia de un asegurador, hagan que crezca; ya que si hay algo que nos ha recordado esta tragedia declarada pandemia, es que desarrollo y bienestar individual, no puede ser alcanzado sin la previa consideración y atención del interés colectivo. El primer paso para lograrlo será la observación y cumplimiento de las reglas que se encuentran preestablecidas.

\section{REFERENCIAS BIBLIOGRÁFICAS:}

Código Civil y Comercial de la Nación Argentina (2015) Rubinzal-Culzoni Editores.

STIGLITZ, R. (2016) Derecho de Seguros, Tomo I, II, III, IV. Buenos Aires: Thomson Reuters La Ley.

Tribunal Supremo, España, Sala Civil, 12/4/2004, R.A. 2612/2004, en Revista de Derecho Mercantil, nro. 254, setiembre-diciembre, (2004). 